COPYRIGHT NOTICE

UB ResearchOnline http://researchonline.ballarat.edu.au

This the accepted version of Global minimizer of large scale stochastic rosenbrock function: canonical duality approach.

The final publication is available at link.springer.com and via

http://dx.doi.org/10.1007/978-3-642-34478-7_82

Copyright 2012 Springer Science+Business Media 


\title{
Global Minimizer of Large Scale Stochastic Rosenbrock Function: Canonical Duality Approach
}

\author{
Chaojie $\mathrm{Li}^{1}$, David Y. Gao ${ }^{1}$, Tingwen Huang ${ }^{2}$, Chuandong $\mathrm{Li}^{3}$, \\ ${ }^{1}$ School of Science, Information Technology and Engineering, \\ University of Ballarat, Mt Helen,VIC 3350, Australia \\ 2 Texas A\&M University at Qatar, Doha, P.O.Box 23874, Qatar \\ 3 College of Computer Science, Chongqing University, Chongqing, 400030, PR China
}

\begin{abstract}
Canonical duality theory for solving the well-known benchmark test problem of stochastic Rosenbrock function is explored by two canonical transformations. Global optimality criterion is analytically obtained, which shows that the stochastic disturbance of these parameters could be eliminated by a proper canonical dual transformation. Numerical simulations illustrate the canonical duality theory is potentially powerful for solving this benchmark test problem and many other challenging problems in global optimization and complex network systems.
\end{abstract}

\section{Preliminary}

Almost all of benchmark test problems in previous literature are deterministic by parameters. However, it is usually more difficult for algorithms to deal with stochastic functions. In Yang's work [2], a stochastic parameter is introduced in Rosenbrock's function such that this well-known benchmark test problem can be proposed as

$$
(\mathcal{P}): \min \left\{P(X)=\sum_{i=1}^{n-1}\left[\left(x_{i}-1\right)^{2}+100 \epsilon_{i}\left(x_{i+1}-x_{i}^{2}\right)^{2}\right] \mid X \in \mathcal{X}\right\},
$$

where $X=\left\{x_{i}\right\} \in \mathcal{X}=\mathbb{R}^{n}$ is a real unknown vector, and the random parameters $\left\{\epsilon_{i}\right\}$ are drawn from a uniform distribution in $[0,1]$. For stochastic functions, most deterministic algorithms such as hill climbing and Nelder-Mead downhill simplex method would simply fail.

\section{Canonical dual approach}

Following the standard procedures in the canonical dual transformation (see Gao, 2009), we first introduce the so-called geometrical operator $\xi=\Lambda(X)$ : $\mathcal{X} \rightarrow \mathcal{E}_{a} \subset \mathbb{R}^{n-1}$

$$
\xi=\left\{\xi_{k}\right\}=\epsilon_{k}^{\frac{1}{2}}\left(x_{k}^{2}-x_{k+1}\right),
$$


and a canonical function $V(\xi)=100 \sum_{k=1}^{n-1} \xi_{k}^{2}$ such that the duality relation

$$
\varsigma=\left\{\varsigma_{k}\right\}=\left\{\frac{\partial V\left(\xi_{k}\right)}{\partial \xi_{k}}\right\}=\left\{200 \xi_{k}\right\}
$$

is invertible. Thus, we have

$$
\xi_{k}=\frac{1}{200} \varsigma_{k} \forall k=1, \ldots, n-1
$$

and the conjugate function of $V\left(\xi_{k}\right)$ can be obtained uniquely by the Legendre transformation

$$
\begin{aligned}
V^{*}(\varsigma) & =\sum_{k=1}^{n-1} \xi_{k} \varsigma_{k}-V(\xi) \\
& =\sum_{k=1}^{n-1}\left\{\xi_{k} \varsigma_{k}-100 \xi_{k}^{2}\right\}=\sum_{k=1}^{n-1} \frac{1}{400} \varsigma_{k}^{2} .
\end{aligned}
$$

Then, the total complementary function can be defined as

$$
\begin{aligned}
\Xi(X, \varsigma) & =\sum_{k=1}^{n-1}\left(x_{k}-1\right)^{2}+\Lambda(X)^{T} \varsigma-V^{*}(\varsigma) \\
& =\sum_{k=1}^{n-1}\left[\left(x_{k}-1\right)^{2}+\epsilon_{k}^{\frac{1}{2}}\left(x_{k}^{2}-x_{k+1}\right) \varsigma_{k}-\frac{1}{400} \varsigma_{k}^{2}\right]
\end{aligned}
$$

For a fixed $\varsigma$ in the canonical dual feasible space $\mathcal{S}_{a} \subset \mathbb{R}^{n-1}$ defined by

$$
\mathcal{S}_{a}=\left\{\varsigma \in \mathcal{S} \mid \epsilon_{k}^{\frac{1}{2}} \varsigma_{k}+1 \neq 0, \quad \forall k=1, \ldots, n-2, \quad \varsigma_{n-1}=0\right\},
$$

the criticality condition $\nabla_{X} \Xi(X, \varsigma)=0$ leads to the following analytical solution

$$
X=\left\{x_{k}\right\}=\left\{\frac{\epsilon_{k-1}^{\frac{1}{2}} \varsigma_{k-1}+2}{2\left(\epsilon_{k}^{\frac{1}{2}} \varsigma_{k}+1\right)}\right\}
$$

Substituting this result into the total complementary function $\Xi(X, \varsigma)$, the canonical dual problem can be finally formulated as

$$
\left(\mathcal{P}^{d}\right): \quad P^{d}(\varsigma)=\max \left\{n-1-\sum_{k=1}^{n-1}\left[\frac{\left(\epsilon_{k-1}^{\frac{1}{2}} \varsigma_{k-1}+2\right)^{2}}{4\left(\epsilon_{k}^{\frac{1}{2}} \varsigma_{k}+1\right)}+\frac{1}{400} \varsigma_{k}^{2}\right] \mid \varsigma \in \mathcal{S}_{a}^{+}\right\},
$$

where

$$
\mathcal{S}_{a}^{+}=\left\{\varsigma \in \mathcal{S}_{a} \mid \epsilon_{k}^{\frac{1}{2}} \varsigma_{k}+1>0, \quad \forall k=1, \ldots, n-2\right\}
$$


By introducing $G(\varsigma), F(\varsigma)$ and $\mathcal{S}_{a}^{+}$such that

$$
\begin{aligned}
& G(\varsigma)=\left[\begin{array}{llll}
\epsilon_{1}^{\frac{1}{2}} \varsigma_{1}+1 & & & \\
& 2\left(\epsilon_{2}^{\frac{1}{2}} \varsigma_{2}+1\right) & & \\
& & \ldots & \\
& & 2\left(\epsilon_{n-2}^{\frac{1}{2}} \varsigma_{n-2}+1\right) & \\
& & & 2
\end{array}\right] \\
& F(\varsigma)=\left[\begin{array}{c}
1 \\
\epsilon_{1}^{\frac{1}{2}} \varsigma_{1}+2 \\
\cdots \\
\epsilon_{n-3}^{\frac{1}{2}} \varsigma_{n-3}+2 \\
\epsilon_{n-2}^{\frac{1}{2}} \varsigma_{n-2}+2
\end{array}\right]
\end{aligned}
$$

We have the following theorem (see Gao, 2009)

Theorem 1. If $\bar{\varsigma}$ is a critical point of $\left(\mathcal{P}^{d}\right)$, then the vector

$$
\bar{X}=G^{-1}(\bar{\varsigma}) F(\bar{\varsigma})
$$

is a critical point of $(\mathcal{P})$ and

$$
P(\bar{X})=P^{d}(\bar{\varsigma}) .
$$

If $\bar{\varsigma} \in \mathcal{S}_{a}^{+}$, then $\bar{\varsigma}$ is the global maximizer of the canonical dual problem $\left(\mathcal{P}^{d}\right)$ on $\mathcal{S}_{a^{+}}$. The vector $\bar{X}$ is a global minimal to the primal problem, and

$$
P(\bar{X})=\min _{X \in \mathcal{X}} P(X)=\max _{\varsigma \in \mathcal{S}_{a}^{+}} P^{d}(\varsigma)=P^{d}(\bar{\varsigma}) .
$$

The proof of this Theorem can be intuitively derived from the paper by Gao (2003).

\section{An Alternative Transformation}

In this section, we choose an alternative canonical dual transformation for stochastic function, which shows analytically that the stochastic perturbation of this problem would never change the global minimal elements.

Let $\xi=\left\{\xi_{k}\right\}=\left\{x_{k}^{2}-x_{k+1}\right\} \in \mathbf{R}^{n-1}$. The canonical function $V(\xi)$ has the form of

$$
V(\xi)=100 \sum_{k=1}^{n-1} \epsilon_{k} \xi_{k}^{2}
$$


Thus, the associated canonical dual variable $\varsigma=\left\{\varsigma_{k}\right\}=\nabla V(\xi)=\left\{200 \epsilon_{k} \xi_{k}\right\}$ and

$$
V^{*}(\varsigma)=\sum_{k=1}^{n-1} \frac{1}{400 \epsilon_{k}} \varsigma_{k}^{2}
$$

Correspondingly, the total complementary function can be written as

$$
\Xi(X, \varsigma)=\sum_{k=1}^{n-1}\left[\left(x_{k}-1\right)^{2}+\left(x_{k}^{2}-x_{k+1}\right) \varsigma_{k}-\frac{1}{400 \epsilon_{k}} \varsigma_{k}^{2}\right] .
$$

By which, the second type of the canonical dual problem can be formulated as

$$
\left(\mathcal{P}^{d}\right): P^{d}(\varsigma)=\max \left\{\left\{n-1-\frac{1}{2} F(\varsigma)^{T} G^{-1}(\varsigma) F(\varsigma)-\frac{1}{400 \epsilon} \varsigma^{T} \varsigma \mid \varsigma \in \mathcal{S}_{a}^{+}\right\}\right.
$$

where

$$
\begin{aligned}
& G(\varsigma)=\left[\begin{array}{llll}
\varsigma_{1}+1 & & & \\
& 2\left(\varsigma_{2}+1\right) & & \\
& & \cdots & \\
& & 2\left(\varsigma_{n-2}+1\right) & \\
& & &
\end{array}\right] \\
& F(\varsigma)=\left[\begin{array}{c}
1 \\
\varsigma_{1}+2 \\
\cdots \\
\varsigma_{n-3}+2 \\
\varsigma_{n-2}+2
\end{array}\right] \\
& \mathcal{S}_{a}^{+}=\left\{\varsigma \in \mathbb{R}^{n-1} \mid \varsigma_{k}>-1, \quad \forall k=1, \ldots, n-2, \quad \varsigma_{n-1}=0\right\} .
\end{aligned}
$$

Theorem 1 still holds for this second canonical dual problem. However, by numerical experiments we can see that the stochastic perturbation does not have any impact on the global optimal solution.

\section{Illustration}

In this section we list some numerical examples with different dimensions, which are more general than normal Rosenbrock function.

Example 1. Consider

$$
(\mathcal{P}): \min \left\{P(X)=\sum_{i=1}^{3}\left[\left(x_{i}-1\right)^{2}+100 \epsilon_{i}\left(x_{i+1}-x_{i}^{2}\right)^{2}\right] \mid X \in \mathcal{X}\right\} .
$$

This problem has the global minimum of all ones and a local minimum near $\left(x_{1}, x_{2}, x_{3}, x_{4}\right)=(-1,1,1,1)$. Correspondingly, the canonical dual problem is

$$
\max \left\{P^{d}(\varsigma)=3-\sum_{k=1}^{3}\left[\frac{\left(\epsilon_{k-1}^{\frac{1}{2}} \varsigma_{k-1}+2\right)^{2}}{4\left(\epsilon_{k}^{\frac{1}{2}} \varsigma_{k}+1\right)}+\frac{1}{400} \varsigma_{k}^{2}\right] \mid \varsigma \in \mathcal{S}_{a}^{+}\right\}
$$


where $\mathcal{S}_{a}^{+}$is defined by (9).

And an alternative canonical dual problem is

$$
\max \left\{P^{d}(\varsigma)=3-\sum_{k=1}^{3}\left[\frac{\left(\varsigma_{k-1}+2\right)^{2}}{4\left(\varsigma_{k}+1\right)}+\frac{1}{400 \epsilon_{k}} \varsigma_{k}^{2}\right] \mid \varsigma \in \mathcal{S}_{a}^{+}\right\}
$$

where $\mathcal{S}_{a}^{+}$is defined by (21). Obviously, it is easy to find results by Matlab optimization tools FMINCON. Note that $\varsigma_{3}=0$, the contour of dual problem can be obtained directly(see Fig. 1). Thus, the global maximum of dual problem is $\left(\varsigma_{1}, \varsigma_{2}, \varsigma_{3}\right)=(0,0,0)$ and the global minimum of primal problem is $\left(x_{1}, x_{2}, x_{3}, x_{4}\right)=(1,1,1,1)$.
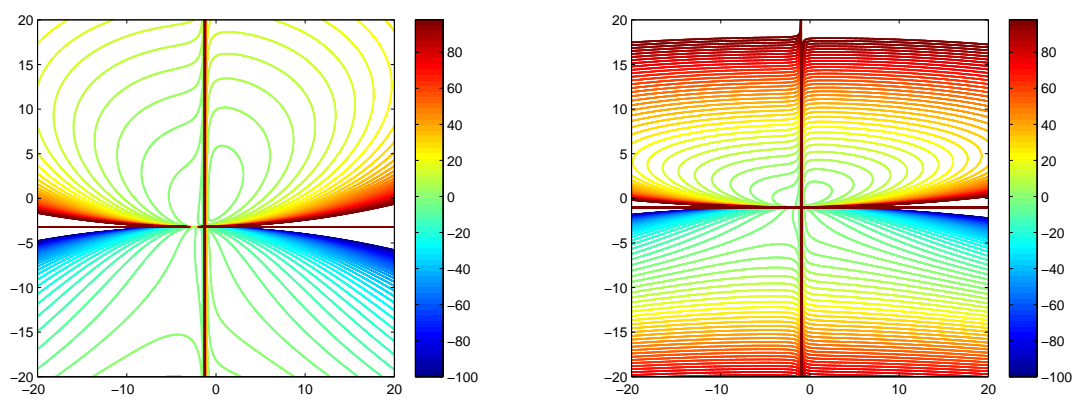

Fig. 1. (a) Dual problem $n=3$ determined by (24) ; (b) Dual problem $n=3$ determined by $(25)$

Example 2. Consider $\mathrm{N}=1000,3000,5000,10000,20000$. The global minimum is inside a long, narrow, banana shaped flat valley. In this case, it is difficult to solve exactly the primal problem $(\mathcal{P})$ by gradient methods. Fortunately, the canonical dual problem is concave maximization over a cone, which can be solved easily, fast and exactly by gradient method.

For (22) and (23), the initial points are chosen randomly from -5 to 5 with the constraints (9) and (21), respectively. With these numerical computation settings, L-BFGS method can quickly solve all these test problems and accurately converge to the global maximizer $\varsigma=(0,0, \ldots, 0)$ with the optimal value $P^{d}(\varsigma)=0\left(10^{-8}\right)$.

All of numerical experiments have been carried out in Intel(R)Core i5-2430M @2.40GHz Windows 7 Home Basic personal notebook computer.

\section{Conclusion}

This paper illustrates that the well-known benchmark test problem of Rosenbrock function with stochastic parameters can be easily solved by the canonical 
duality theory. Numerical examples show that even though the nonconvex primal problem has been disturbed by stochastic parameters, the canonical duality theory can avoid defective influence to achieve global optimal solution stably. The canonical duality theory can be used for solving some more challenging problems in global optimization and complex network systems.

\section{Acknowledgements}

This research is supported by US Air Force Office of Scientific Research under the grant AFOSR FA9550-10-1-0487 and by Australia Government grant through the Collaborative Research Network(CRN) to the university of Ballarat.

\section{References}

[1] Rosenbrock, H.H.: An automatic method for finding the greatest or least value of a function. The Computer Journal 3: 175184.(1960)

[2] Yang X. S.,Deb S.: Engineering optimization by cuckoo search, Int. J. Math. Modelling Num. Optimisation, Vol. 1, No. 4, 330-343 (2010)

[3] Gao, D.Y.: Duality, triality and complementary extremum principles in nonconvex parametric variational problems with applications. IMA J. Appl. Math. 61, $199235(1998)$

[4] Gao, D.Y.: Pure complementary energy principle and triality theory in finite elasticity. Mech. Res. Commun. 26(1), 3137 (1999)

[5] Gao, D.Y.: General analytic solutions and complementary variational principles for large deformation nonsmooth mechanics. Meccanica 34, 169198 (1999)

[6] Gao, D.Y.: Duality Principles in Nonconvex Systems: Theory, Methods and Applications. Kluwer Academic, Dordrecht (2000)

[7] Gao, D.Y.: Canonical dual transformation method and generalized triality theory in nonsmooth global optimization. J. Glob. Optim. 17(1/4), 127160 (2000)

[8] Gao, D.Y.: Perfect duality theory and complete solutions to a class of global optimization problems. Optim. 52(4-5), 467-493(2003)

[9] Gao, D.Y.: Perfect duality theory and complete solutions to a class of global optimization problems. Optimization 52(45), 467493 (2003)

[10] Gao, D.Y.: Nonconvex semi-linear problems and canonical dual solutions. In: Gao, D.Y., Ogden, R.W. (eds.) Advances in Mechanics and Mathematics, vol. II, pp. 261312. Kluwer Academic, Dordrecht (2003)

[11] Gao, D.Y.: Canonical duality theory and solutions to constrained nonconvex quadratic programming. J. Glob. Optim. 29, 377399 (2004)

[12] Gao, D.Y.: Solutions and optimality to box constrained nonconvex minimization problems. J. Ind. Manag. Optim. 3(2), 293304 (2007)

1. Gao, D.Y.: Canonical duality theory: theory, method, and applications in global optimization. Comput. Chem. 33, 1964-1972, (2009)

[13] Gao, D.Y. and Wu, C.Z.: On the triality theory in global optimization. J. Global Optimization (2010)

[14] Gao, D.Y, Zhang J.P: Canonical Duality Theory for Solving Minimization Problem of Rosenbrock Function. arxiv.org/abs/1108.1241v1 